\title{
Methode zur Untersuchung der Lernfähigkeit von Kälbern in Gruppenhaltung und Ergebnisse bei visuellen Differenzierungs- aufgaben
}

\section{Summary}

Title of the paper: Method to assess the learning ability of group-housed calves and results of visual discrimination tasks

Assessing the sensory capacity and learning ability of farm animals will be of increasing importance for the evaluation of animal welfare in order to develop new efficient farming technologies which seriously consider the animals' well-being.

The more-arm-maze test using optic signalling proved to be a useful method to assess the learning ability of singel or group-housed calves. Each test box consisted of 3 stands per calf arranged side by side (stand dimensions: $1.00 \mathrm{~m}$ long, $0.30 \mathrm{~m}$ wide and $1.50 \mathrm{~m}$ high, wooden walls). The stands could be distinguished by coloured lamps or black visual patterns of different shapes, projected on a white plastic pancl above the entrance as stimuli. Chosing the right (conditioned) stimulus the calves were rewarded by milk (unconditioned stimulus) when suckling at the dispenser.

Given a random expectation of $33 \%$, the calves reached at least $70 \%$ correct choices already at the 4 th test day.

Key words: learning, device, visual discrimination, cattle, calf, group-housing

\section{Zusammenfassung}

Die Prüfung der sensorischen und kognitiven Fähigkeiten von Nutztieren erhält sowohl im Hinblick auf eine bessere Beurteilung ihrer Befindilichkeit als auch für die Entwicklung neuer, automatisierter Tierversorgungsanlagen eine zunehmende Bedeutung.

Die mit einem Mehrkanalwahlverfahren durchgeführten Versuche lassen den Schluß zu, daß die Prüfung der Lernfähigkeit von einzelnen oder in kleinen Gruppen gehaltenen Kälbern damit möglich ist. In Testboxen waren nebeneinander je Kalb 3, aus Spanplatten völlig gleich gestaltete Stände mit den Abmessungen 1,0 m Länge, 0,3 $\mathrm{m}$ Breite und 1,5 m Höhe eingerichtet. Durch die Abmessungen war gewährleistet, daß sich in einem Stand nur jeweils ein Kalb aufhalten konnte. Die Stände unterschieden sich durch Signallampen, die entweder verschiedene Farben oder verschiedene schwarz / weiß Symbole wiedergaben. In dem Stand mit dem belohnten Stimulus $(\mathrm{S}+)$ konnten die Kälber aus dem an der Stirnwand angebrachten Sauger Milch aufnehmen. In den übrigen Ständen (S-) waren die Sauger nicht an die Milchversorgung angeschlossen.

Bei einer Zufallswahrscheinlichkeit von $33 \%$ für S+ erreichten die Kälber bereits am 4. Tag einen Anteil richtiger Entscheidungen von über $70 \%$.

Schlüsselwörter: Lernen, Apparatur, visuelle Differenzierung, Rind, Kalb, Gruppenhaltung

\section{Einleitung}

Die richtige Beurteilung der Lernfähigkeit von Nutztieren und die Ableitung entsprechender Schlußfolgerungen aus ihrem Lernverhalten war schon immer eine wichtige Aufgabe des Tierhalters und Tierzüchters.

Mit der zunehmenden Arbeitsteilung zwischen den Entwicklern der verschiedensten 
Haltungstechniken einerseits sowie Tierbetreuern und Tierbesitzern andererseits wurden die aus der Tierbetreuung erhältlichen Rückinformationen für die permanente Optimierung von Tierhaltungen eingeschränkt. Daraus können sich Fehleinschätzungen ergeben, die bei besserer Beurteilung des Verhaltens, insbesondere des Lernverhaltens, vermeidbar wären.

Die Forschung hat sich mit der Lernfähigkeit von Nutztieren bisher relativ wenig befaßt. Dagegen wurde die Lernfähigkeit von Zoo- und Labortieren intensiv untersucht. Für den Zeitraum von 1950 - 1996 kann man über 80000 Arbeiten zum Lernverhalten von Zoo- und Labortieren und nur etwa $200 \mathrm{zu}$ dem von Nutztieren recherchieren (KILGOUR, 1976; PORZIG u.a., 1978; KILGOUR, 1987; KILGOUR u.a., 1991; ARAVE, 1996).

Der größte Anteil der Lernforschung mit Labortieren war medizinischen und pharmazeutischen Fragestellungen gewidmet. Dabei lieferten die unterschiedlichsten Lerntests wertvolle Informationen über komplexe Interaktionen und für die Aufdeckung von Kausalbeziehungen. In entsprechender Weise könnte auch die Forschung zur Biologie landwirtschaftlicher Nutztiere durch vergleichende Beurteilung des Lernverhaltens zu Erkenntnissen bezüglich der Befindlichkeit und der sensorischen und kognitiven Fähigkeiten gelangen. Das erscheint aus verschiedenen Gründen auch notwendig. Einerseits ist die Entwicklung der Tierbetreuung durch einen Trend zur Automatisierung gekennzeichnet. Für die Entwicklung von automatisierten Fütterungs-, Pflege- und Melkanlagen erscheint die Kenntnis der sensorischen und kognitiven Fähigkeiten der betreffenden Tiere zweckmäßig. Andererseits könnte ein besseres Allgemeinwissen über die so vielfältig genutzten Tiere zu einer Verbesserung der Diskussion wichtiger Tierschutzfragen und dadurch zu einer verbesserten Tierhaltung führen.

Gegenwärtig gibt es in Europa schon über 70 Milchproduktionsbetriebe, in denen Kühe durch vollautomatische Melksysteme und Kraftutterautomaten versorgt werden. Dabei werden Signale zur Steuerung des Verhaltens der Kühe kaum genutzt. Es fehlt möglicherweise der wissenschaftliche Vorlauf dafür. Eine Übersicht zu den bisherigen wissenschaftlichen Publikationen über Lernexperimente mit Rindern wird in der Tabelle 1 gegeben. Von den 33 Untersuchungen waren 13 auf die Wahrnehmung visueller Reize und 5 auf die von akustischen Reizen ausgerichtet. Alle Autoren, die Farben in das Lernparadigma einbezogen hatten, kamen zu dem Schluß, daß Rinder Farben unterscheiden können (LAUBE, 1975; THINES und SOFFIE, 1977; GRÄF und SAMBRAUS, 1978; SOFFIE u.a., 1980; DABROWSKA u.a., 1981; GILBERT und ARAVE, 1986; RIOL u.a., 1989; ARAVE u.a., 1993). Offen blieb jedoch dabei die Frage nach der Art des Farbsehvermögens, danach, ob Rinder di-, tri- oder polychromatisch sehen.

Wichtige Erkenntnisse wurden mit Experimenten erzielt, in denen verschiedene Futterzusätze oder verschiedene Futterkonservierungsarten durch das Wahlverhalten der Tiere beurteilt wurden (KLOPFER u.a., 1981; ARAVE u.a., 1985, 1988, 1990, 1991; WIEDMEIER u.a., 1994).

Das Wahlverhalten von Nutztieren wurde ebenfalls mit sehr gutem Erfolg für die Einschätzung verschiedener Haltungsbedingungen eingesetzt (WANDER, 1976; PIO- 
Tabelle 1

Publikationen über Lernexperimente mit Rindern (Publications about learning ability of cattle)

\begin{tabular}{|c|c|c|c|c|c|c|}
\hline $\begin{array}{l}\text { Erst-Autor, } \\
\text { Jahr }\end{array}$ & Tiere & Anzahl & $\begin{array}{l}\text { Test } \\
\text { Einzel/Gruppe }\end{array}$ & $\begin{array}{l}\text { Wahl- } \\
\text { Apparatur }\end{array}$ & Paradigma & $\begin{array}{l}\text { Erfolgs- } \\
\text { wahrscheinlk }\end{array}$ \\
\hline $\begin{array}{l}\text { Kudryavtzew, } \\
1962\end{array}$ & Kühe & zit.(2) & Einzel & 1 Kanal- & Töne, $1 \mathrm{k} \mathrm{Hz}$ & 0,5 \\
\hline $\begin{array}{l}\text { Albright, } \\
1966\end{array}$ & Kühe & 40 & Gruppe & 1 Kanal- & Töne, & 0,5 \\
\hline $\begin{array}{l}\text { Wieckert, } \\
1966\end{array}$ & Kälber & 20 & Einzel & 2 Kanal- & Schwarz/Weiß & 0,5 \\
\hline $\begin{array}{l}\text { Gramling, } \\
1970\end{array}$ & Kälber & 29 & Einzel & 2 Kanal- & Schwarz/Weiß & 0,5 \\
\hline $\begin{array}{l}\text { Liebenberg, } \\
1971\end{array}$ & Review & 91 Publik. & & & & \\
\hline $\begin{array}{l}\text { Schaeffer, } \\
1971\end{array}$ & Kälber & 24 & Einzel & 2 Kanal- & $\begin{array}{l}\text { Schwarz/Weiß } \\
\text { Groß-/Klein- } \\
\text { Symbol } \\
\end{array}$ & 0,5 \\
\hline Polten, 1974 & Kühe & 8 & Einzel & 1 Kanal- & Töne, $1-3 \mathrm{kHz}$ & - \\
\hline Laube, 1975 & Jungrinder & 4 & Einzel & 2 Kanal- & $\begin{array}{l}\text { Farben/Grau, } \\
\text { Minim. separ. }\end{array}$ & 0,5 \\
\hline Wander, 1976 & Kühe & 29 & Gruppe & 2-fach Wahl & \begin{tabular}{|l|} 
Platz \\
\end{tabular} & 0,5 \\
\hline Kilgour, 1976 & Review & 45 Publik. & & & & \\
\hline Thines, 1977 & Jungrinder & 6 & Einzel & 2- fach Wahl & Farben / Grau & 0,5 \\
\hline Gräf, 1978 & Jungbullen & 6 & Einzel & 2- fach Wahl & Farbe / Grau & 0,5 \\
\hline Porzig, 1978 & Review & 53 Publik. & & & & \\
\hline Porzig, 1979 & Kühe & 7 & Einzel & 1 Kanal- & Töne, $1-3 \mathrm{kHz}$ & - \\
\hline Soffie, 1980 & Jungrinder & 6 u. 24 & Einzel & 2 Tröge & Farben/Grau & 0,5 \\
\hline Kilgour, 1981 & Kühe & 73 & Einzel & Maze (1) & \begin{tabular}{|l|} 
Platz \\
\end{tabular} & 0,5 \\
\hline $\begin{array}{l}\text { Dabrowska, } \\
1981\end{array}$ & Kühe & 11 & Einzel & 2 Tröge & Farben/Grau & 0,5 \\
\hline Klopfer, 1981 & Kühe & 2 & Einzel & 2 Tröge & Futtermittel & 0,5 \\
\hline $\begin{array}{l}\text { Baldwin, } \\
1981 \\
\end{array}$ & Kälber & 3 & Einzel & 2 Schalter & Symbole & 0,5 \\
\hline Heffner, 1983 & Rinder & 2 & Einzel & 1 Schalter & $\begin{array}{l}\text { Töne, } 23 \mathrm{~Hz} \text { - } \\
35 \mathrm{k} \mathrm{Hz}\end{array}$ & 0,5 \\
\hline $\begin{array}{l}\text { Hoonhout, } \\
1984\end{array}$ & Kühe & $\begin{array}{l}\text { Diss. } \\
\text { Waikato, NZ }\end{array}$ & Einzel & 2 Schalter & $\begin{array}{l}\text { Töne, } 1 \text { - } 18 \\
\mathrm{k} \mathrm{Hz}\end{array}$ & 0,5 \\
\hline Arave, 1985 & \begin{tabular}{|l|} 
Jungrinder \\
\end{tabular} & 8 & Einzel & 2 Tröge & \begin{tabular}{|l|} 
Futtermittel \\
\end{tabular} & 0,5 \\
\hline $\begin{array}{l}\text { Kovalcik, } \\
1986\end{array}$ & $\begin{array}{l}\text { Jungrinder u. } \\
\text { Kühe }\end{array}$ & 39 & Einzel & 2 Kanal- & Platz & 0,5 \\
\hline Gilbert, 1986 & Jungrinder & 8 & Einzel & 2 Schalter & $\begin{array}{l}\text { Farben, } \\
\text { monochrom. }\end{array}$ & 0,5 \\
\hline Kilgour, 1987 & Review & 86 Publik. & & & & \\
\hline Purcell, 1988 & Kälber & $\begin{array}{l}\text { Diss.Utah } \\
\text { Uni. }\end{array}$ & Einzel & 2 Kanal- & Platz & 0,5 \\
\hline Arave, 1988 & Jungrinder & 8 & Einzel & 2 Tröge & Futtermittel & 0,5 \\
\hline Riol, 1989 & Kühe & 8 & Einzel & 2 Tröge & Farben/Grau & 0,5 \\
\hline Boissy, 1990 & Jungrinder & 44 & Einzel & 1 Hebel & \begin{tabular}{|l|l|} 
Platz \\
\end{tabular} & - \\
\hline Arave, 1990 & Jungrinder & 8 & Einzel & 2 Tröge & Futterzusätze & 0,5 \\
\hline
\end{tabular}


Tabelle 1 (Fortsetzung)

\begin{tabular}{|l|l|l|l|l|l|l|}
\hline Purcell, 1991 & Jungrinder & 17 & Einzel & 2 Kanal- & Platz & 0,5 \\
\hline Kilgour, 1991 & Review & 254 Publik. & & & & \\
\hline Arave, 1991 & Jungrinder & 8 & Einzel & 2 Futterautom & Futterzusätze & 0,5 \\
\hline Arave, 1992 & Kälber & 275 & Einzel & 2 Kanal- & Platz & 0,5 \\
\hline Entsu, 1992 & Jungrinder & 3 & Einzel & 2 Kanal-. & Symbole & 0,5 \\
\hline Arave, 1993 & Jungrinder & 16 & Einzel & 2 Schalter & Farben, Intensität & 0,5 \\
\hline $\begin{array}{l}\text { Wiedmeier, } \\
1994\end{array}$ & Jungrinder & 8 & Einzel & 2 Futterautom & Futterzusätze & 0,5 \\
\hline Grandin, 1994 & Jungrinder & 12 & Einzel & 2 Kanal- & Platz & 0,5 \\
\hline Arave, 1996 & Review & 84 Publik. & & & & \\
\hline
\end{tabular}

(1) HEBB- WILLIAMS closed field test

(2) zit. bei ALBRIGHT u.a. (1966)

TROWSKI, 1992). Für die tiergerechte Gestaltung von Liegeboxen, von Stallfußböden, von Liegeflächen oder von Mikroklimazonen wurden durch Wahlversuche mit Rindern wertvolle Erkenntnisse gewonnen. Besonders hervorgehoben werden soll, daß WANDER diese Wahlversuche bei Aufrechterhaltung des Sozialkontaktes der Rinder während der Prüfungen gestaltet hat, während fast alle anderen Autoren die Tiere isoliert prüften. Mit einer Prüfung von Individuen innerhalb der für sie geeigneten sozialen Gruppenstruktur entfällt der mit der Isolation verbundene Streß für das Tier und der Einfluß des prüfenden Menschen wird geringer (vgl. WARNICK u.a., 1977).

Die Fähigkeit von Rindern, verschiedene Töne zu differenzieren, wurde in 5 Untersuchungen nachgewiesen (KUDRYAVTZEW, 1962; POLTEN, 1974; PORZIG u.a., 1979; HEFFNER und HEFFNER, 1983; HOONHOUT, 1984). Danach sind Rinder in der Lage, im Frequenzbereich von $1-35 \mathrm{k} \mathrm{Hz}$ Töne zu unterscheiden.

Insgesamt ergibt sich aus den in der Recherche erfaßten, relativ wenigen Untersuchungen zur Lernfähigkeit von Rindern der Eindruck, daß dieser Tierart durch die Lernforschung bisher nicht angemessen entsprochen wurde. Eine mögliche Ursache dafür könnte neben dem hohen finanziellen Aufwand derartiger Experimente in dem Mangel an geeigneten Methoden bestehen. Wie aus der Tabelle 1 ersichtlich ist, wurde bisher fast nur mit dem Einzeltest gearbeitet. Das ist sehr arbeits- und zeitaufwendig. Außerdem stellt die Isolation von Tieren in der Testsituation einen Störfaktor dar, da in der modernen Rinderhaltung die Gruppenhaltung die Regel ist. Die für den Einzeltest notwendige Isolation führt zu Verhaltensänderungen (Umherlaufen, Brüllen, u.a.), die das Lernverhalten, individuell unterschiedlich, beeinträchtigen können. Schließlich sind nur bei Gruppentests Untersuchungen der Wechselwirkungen zwischen dem Lernverhalten und dem Sozialverhalten möglich.

Das Ziel der Arbeit bestand daher darin, eine für Rinder geeignete, neue Lernapparatur zu entwickeln und Möglichkeiten für den Gruppentest zu zeigen. Dabei war zu sichern, daß die Tiere sich an der Lernapparatur nicht gegenseitig bei ihrem Wahlverhalten behindern und möglichst nicht durch Vorbildwirkung beeinflussen konnten. Diesen Forderungen sollte durch eine vom Tier freiwillig gewählte, geeignete Vereinzelung innerhalb von Gruppenboxen entsprochen werden. Es war zu prüfen, ob bestimmte Präferenzen, beispielsweise eine Seitenstetigkeit, das Lernergebnis beeinflussen und ob die Rinder andere Erkennungsmöglichkeiten für die Stände, in denen eine Belohnung 
zu erhalten war, nutzten. Mit der zu entwickelnden Methode sollte die Fähigkeit von Kälbern zur Differenzierung der Farben Gelb, Grün, Rot und Blau sowie von Schwarz/ Weiß-Mustern (Druckbuchstaben A und O ) geprüft werden.

\section{Methodik und Tiere}

In Testboxen waren nebeneinander je Tier drei völlig gleich gestaltete Stände von 0,3 $\mathrm{m}$ Breite, 1,0 m Länge und 1,5 m Höhe aus weiß beschichteten Spanplatten eingerichtet (Draufsicht in Abb. 1). An der Vorderwand der Stände waren die den Kälbern bekannten Sauger für Milch angebracht. Ein Stand war mit dem belohnten Stimulus (S+) und zwei Stände mit dem nicht belohnten Stimulus (S-) gekennzeichnet, so daß sich eine zufällige Erfolgswahrscheinlichkeit für $\mathrm{S}+$ von $\mathrm{p}=0,33$ ergab. In den Ständen mit dem S+ Stimulus konnten die Kälber über die Sauger Milch ad libitum aufnehmen, in den übrigen Ständen waren die Sauger nicht an die Milchversorgung angeschlossen. Die Zuordnung der S+ und S- Stimuli zu den einzelnen Ständen wurde täglich zur Mittagszeit gewechselt. Durch die Standabmessungen war gewährleistet, daß nur ein Kalb einen Stand betreten und sich darin ungestört aufhalten konnte. Die Stände unterschieden sich nur durch die visuellen Stimuli (S+ u. S-). Diese wurden durch Signallampen dargestellt, die entweder verschiedene Farben oder verschiedene Schwarz / Weiß - Muster zeigten. Diese Signallampen, handelsübliche Wärmestrahllampen mit einem quadratischen Vorsatzrahmen $(22 \times 22 \mathrm{~cm})$ für die Folien, waren oberhalb der Stände so angebracht, daß sie von den Kälbern gut zu sehen, aber nicht zu erreichen waren (1,40 m hoch, 0,40 m vom Eingang des Standes entfernt, schräg nach hinten gerichtet). Für die Projektion der Schwarz / Weiß - Muster wurden Polylux- Folien in einen Rahmen gespannt und durch eine 60 Watt- Glühbirne beleuchtet. Als Schwarz I Weiß - Muster wurden die Druckbuchstaben A und O verwandt (Höhe $15 \mathrm{~cm}$, Strichbreite $18 \mathrm{~mm}$ ).

Für die Darstellung der Farben Gelb, Grün, Blau und Rot wurden 60 Watt- Spotline Disco - Glühbirnen der Firma Philips ( made in E.C., $230 \mathrm{~V}$ ) verwandt.

Für die Kälber bestand zweimal täglich für 1,5 Stunden freie Wahlmöglichkeit. In der restlichen Zeit waren die Stände geschlossen. Während der Öffnungszeit konnten sie in den mit dem S+ Stimulus gekennzeichneten Ständen, nach der Abbildung 1 beispielsweise in den Ständen 4 und 6, Milch aufnehmen.

Jeder Standbesuch wurde durch Videoaufzeichnung erfaßt. Der Lernerfolg im Tagesverlauf(LT) wurde als der prozentuale Anteil Besuche in Ständen mit dem S+ Stimulus an den Gesamtbesuchen des Tages definiert.

Es wurden schwarzbunte männliche Kälber aus dem Dummerstorfer Rinderbestand in die Lerntests einbezogen, die in der ersten Lebenswoche in den Versuchsstall eingestellt worden waren. Sie wurden in Gruppenboxen auf Stroh gehalten, hatten Selbsttränken für Wasser sowie Heu ständig zur Verfügung und wurden mit Milch semi ad libitum getränkt. Ihre tägliche Aufnahme lag bei 10 - 12 Liter Milchaustauschertränke (Fa. Salvana, AM7, 13\% TS).

Zur Prüfung methodischer Fragen wurden vor den eigentlichen Lerntests bzw. mit anderen Kälbern zeitgleich zum Lerntest drei Versuche durchgeführt. 


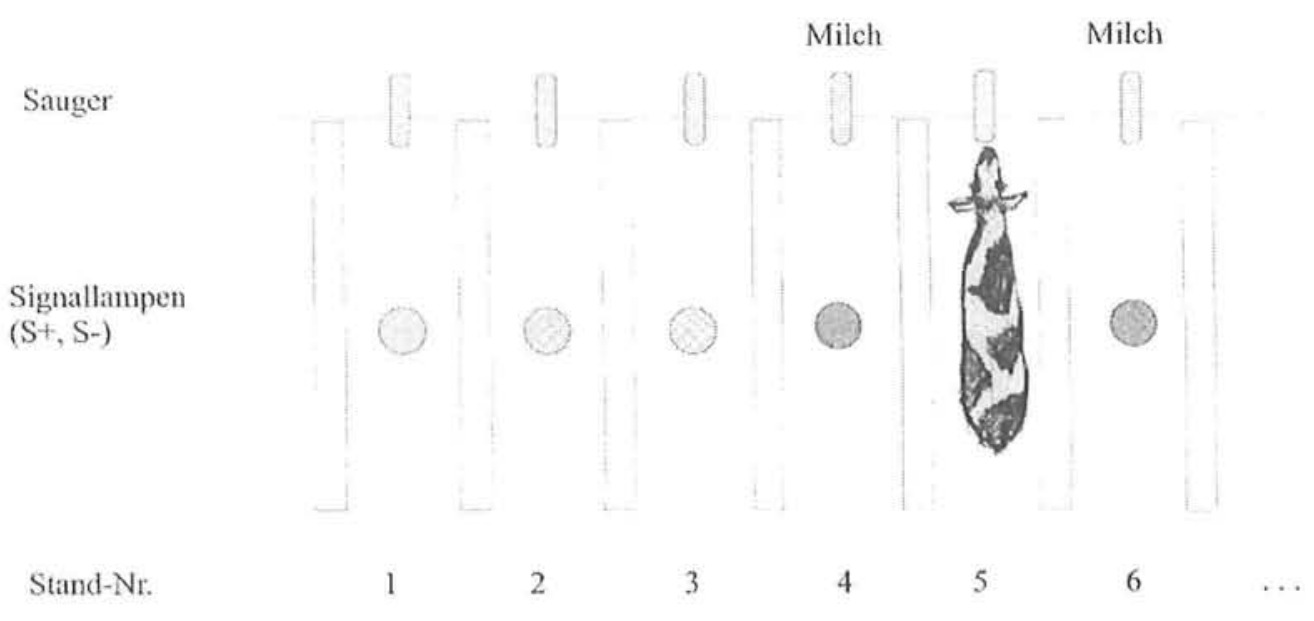

täglicher Wechsel der Position der S+ Stimuli, im Bild Stand 4 und 6

Abb. 1: Mehrkanal - Wahlanlage für Kälber (Draufsicht) (More-arm - maze for calves)

Im Versuch 1 wurde die individuelle Aktivität bezüglich der Anzahl von Standbesuchen von Kälbern in elektronisch kontrollierten Ständen über 48 Stunden geprüft. Diese Prüfung erfolgte im Gruppenmittel am 14./15. Lebenstag. Jeweils zwei benachbarte Kälber, deren Testboxen durch ein niedriges Brettergitter getrennt war, konnten in sechs Ständen, 3 je Kalb, von 6,30 - $8^{\circ \circ}$ und von $15^{\circ \circ}-16,30 \mathrm{Uhr}$ Milch aufnehmen. In der übrigen Zeit waren die Stände offen und die Sauger nicht an die Milchversorgung angeschlossen.

Im Versuch 2 wurde am 21. Lebenstag (Gruppenmittel) geprüft, ob die Kälber alle Stände gleich häufig besuchten, wenn überall Milch erreichbar war. Wie in der Testsituation bestand zweimal täglich für 1,5 Stunden freie Wahlmöglichkeit. Während dieser Zeiten konnten die Kälber in allen Ständen Milch aufnehmen.

Tabelle 2

Experiment zur Prüfung der Lernfähigkeit schwarzbunter männlicher Kälber (Experiment to assess the learning ability in male calves

\begin{tabular}{lclc}
\hline $\begin{array}{l}\text { Versuchsablauf } \\
\text { Test-Nr. }\end{array}$ & Alter bei Testbeginn (1) & Testart & Testdauer (Tage) \\
\hline 1 & 22 Tage & 1. Lerntest & 6 \\
& & (Signale für S+ / S-: grün / gelb und umgekehrt)(2) & 3 \\
3 & 71 Tage & Erinnerungstest entsprechend Test 1 & 4 \\
4 & 74 Tage & Umlerntest zu rot / blau und umgekehrt & 7 \\
\hline
\end{tabular}

(1) Gruppenmittel

(2) Um eine Beeinflussung der Ergebnisse der Lemtests durch eventuelle Prafferenzen für bestimmte Signale zu vermeiden, wurde die Halfte der Tiere im Test 1 bei grün belohnt, die andere Hälfte bei gelb. Die gleiche counterbalance Technik wurde auch in den Tests 2,3 und 4 angewendet. 
Im Versuch 3 wurde mit 10 Kälbern, die nicht in die weiteren Lerntests einbezogen wurden, geprüft, ob sie andere Erkennungsmöglichkeiten für die Wahl der Stände mit dem S+ Stimulus nutzten, als die Signallampen.

Zur Beurteilung des Erinnerungsvermögens der Kälber waren als Kontrolle zum Erinnerungstest 6 naive, gleichaltrige Kälber geprüft worden, die nicht in die übrigen Lerntests einbezogen wurden.

In die eigentlichen Lernexperimente waren 16 Kälber einbezogen worden. Diese Kälber wurden während der Testzeiten in Gruppen zu 4 Tieren gehalten. Das Alter bei Testbeginn und die jeweiligen Wahlaufgaben sind aus der Tabelle 2 ersichtlich.

\section{Ergebnisse}

Die Tiere unterschieden sich in ihrer Aktivität erheblich. In der Abbildung 2 ist eine entsprechende Klassifizierung dargestellt. Die mittels elektronischer Tiererkennung erfaßten Standbesuche von durchschnittlich zwei Wochen alten Kälbern zeigen, daß die aktivsten Kälber etwa 50 Besuche in 24 Stunden und die inaktiven Kälber nur etwa 5 Besuche machten. Der Median lag bei etwa 20 Besuchen je Tag.

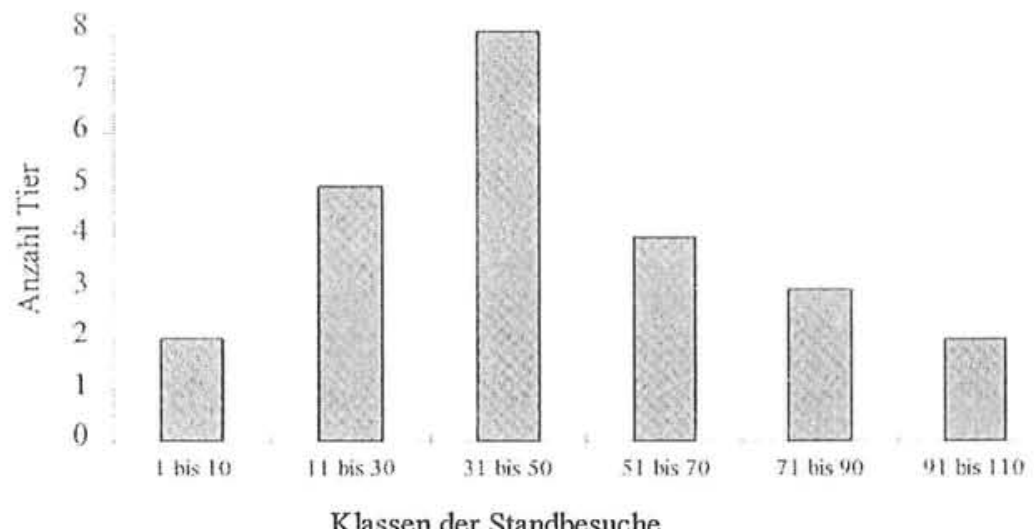

Abb. 2: Häufigkeitsverteilung der Besuche an den Tränkständen pro Tier in 48 Stunden (Versuch 1, 14./15. Lebenstag, $n=24$ ) (Distribution of stand choices in 48 hours)

Eine Prüfung auf möglicherweise bestehende Präferenzen bestimmter Stände durch die Kälber ergab, daß die Kälber innerhalb eines Tages alle Stände etwa zwei bis dreimal besucht haben (s. Abb. 3). Eine Bevorzugung bestimmter Stände wurde nicht beobachtet.

Die Nutzung eventueller anderer Erkennungsmöglichkeiten für die Stände mit der Belohnung, als die Signallampen, wurde mit dem in Abbildung 4 gezeigten Experiment geprüft. 


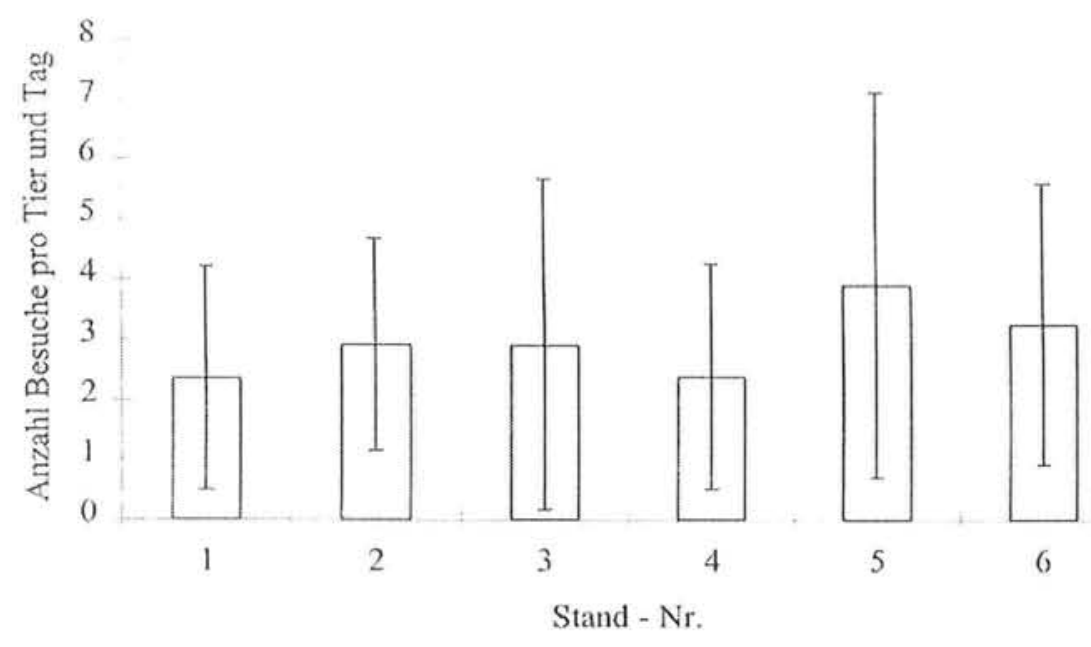

Abb. 3: Verteilung beı der Standwahl durch naive Kälber, Belohnung in allen Ständen (Versuch 2, $\mathrm{n}=23$ ) (Distribution of stand choices of naive calves, rewarding in all stands)

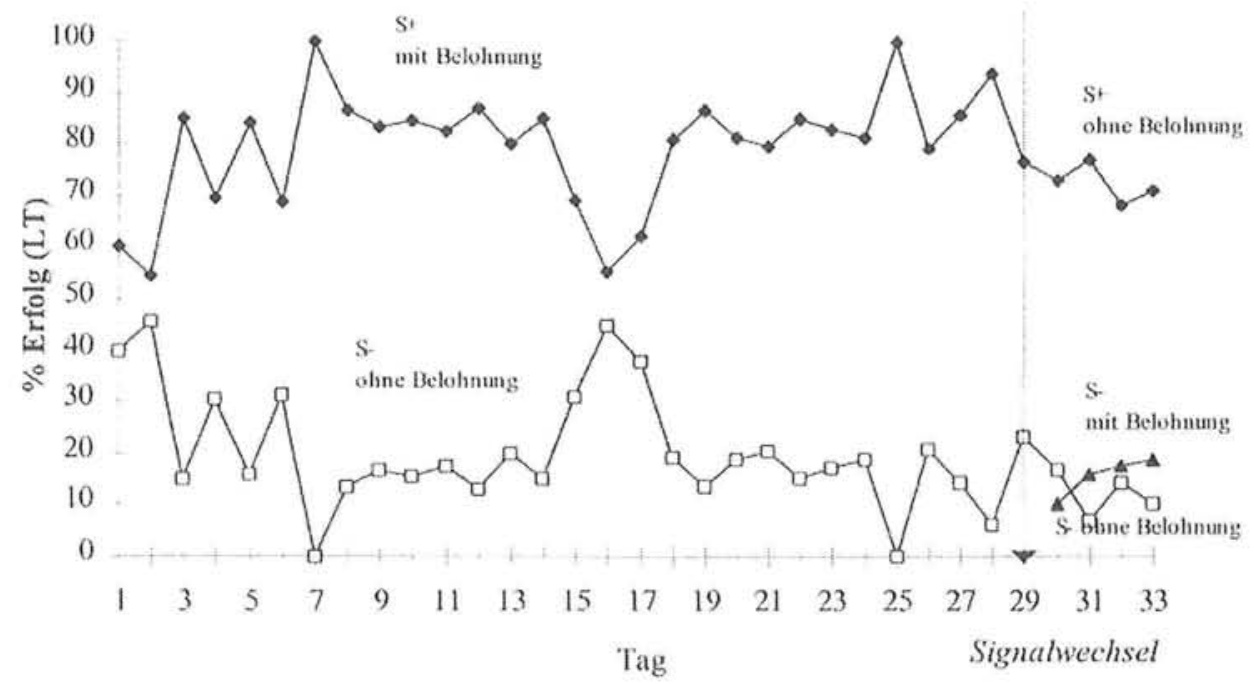

Abb. 4: Standwahl bei Änderung des S+ Stimulus nach dem 29. Tag (Positionswechsel der S+ Stände von Mahlzeit zu Mahlzeit, Versuch 3, $n=10$ ) (Stand choices on consecutive tetdays with change the s+ stimulus after the 29 th day)

Das Verhalten der Kälber bei Änderung des S+ Stimulus nach dem 29. Tag zeigt, daß sie in diesem Zeitraum andere Erkennungsmöglichkeiten für die mit Milch belohnten Stände nicht nutzten. Sie besuchten auch weiterhin zu $70-80 \%$ die mit dem bisherigen S+ Stimulus gekennzeichneten Stände, obwohl die Milch an einem der bisherigen S- Stände erreichbar war und von allen Kälbern auch dort mehrmals am Tage aufge- 
nommen wurde.

In den aufeinander folgenden vier Lerntests war die Aktivität der Kälber, gemessen an der durchschnittlichen Anzahl Standbesuche je Tag, bei neuen Wahlaufgaben (Test 1, 3 u. 4) anfänglich etwas erhöht (s. Abb. 5). Besonders in den Tests 3 und 4 war die Aktivität zu Beginn deutlich erhöht. Die Mittelwerte für die Anzahl Standbesuche lagen bei der zeitlich begrenzten Besuchsmöglichkeit zwischen 6 bis 11 Besuchen je Tier und Tag.

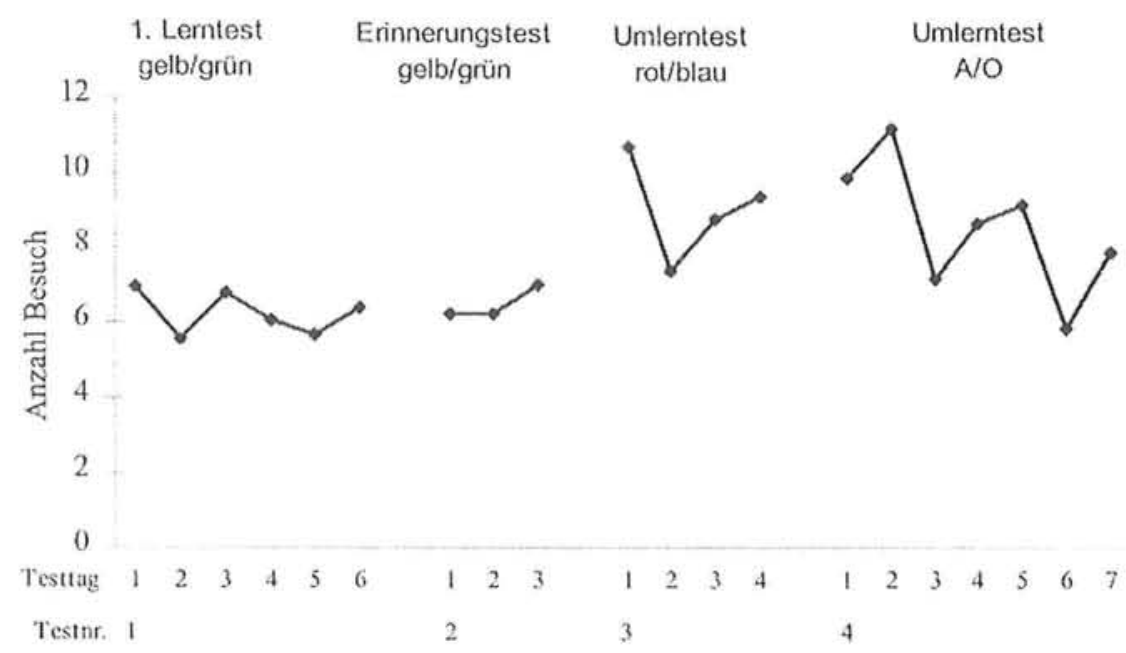

Abb. 5: Durchsclnittliche Anzahl Standbesuche pro Tier und Tag (Number of stand choices per calf and day)

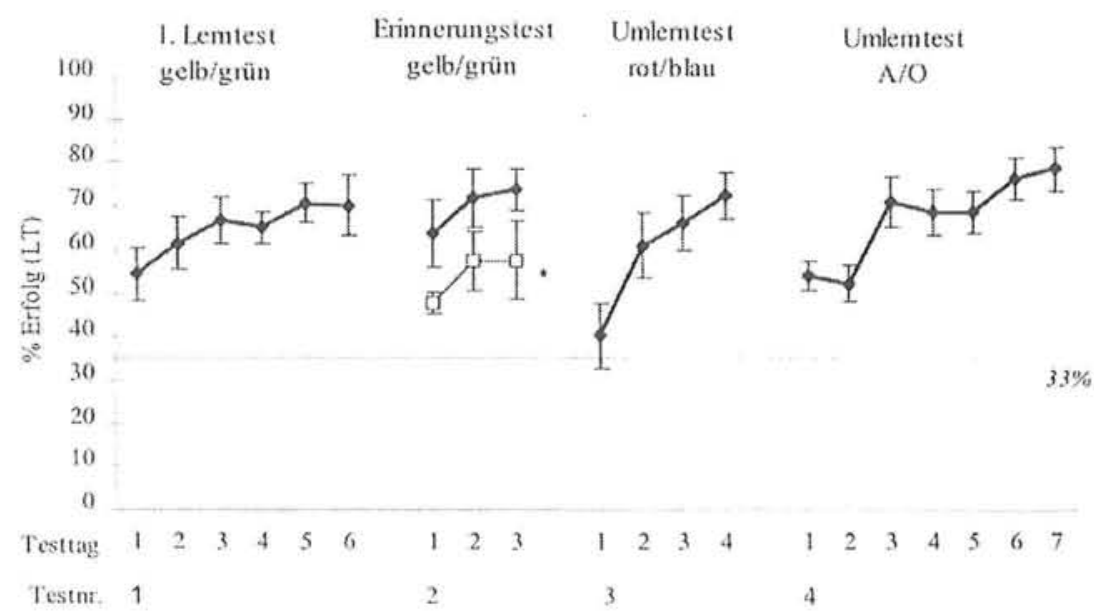

Abb. 6: Anteil richtiger Standbesuche (LT\%) bei aufeinander folgenden Lerntests (* naive Tiere) (Correct stand choices on consecutive testdays (LT\%) in different learning tests) 
Der durchschnittliche Lernerfolg der Kälber ist in Abbildung 6 für die vier Lerntests dargestellt. Der erste Lerntest begann mit dem 22. Lebenstag und dauerte 6 Tage. Die Kälber hatten die Farben Grün und Gelb zu unterscheiden. Bereits am ersten Testtag lagen sie mit über $50 \%$ richtiger Standbesuche signifikant über der zufälligen Erfolgswahrscheinlichkeit von $33 \%$ und erreichten ab 4. Testtag Anteile richtiger Standbesuche von $70-80 \%$.

Nach 49 Tagen folgte ein Erinnerungstest über drei Tage. Die Tiere erreichten dabei am ersten Testtag mit $64 \%$ ein (nicht signifikant) besseres Ergebnis, als sie am ersten Tag im Test 1 mit $55 \%$ erreicht hatten. Die zum Vergleich in diesem Alter erstmals geprüften 6 naiven Kontolltiere ereichten $46 \%$ am ersten Testtag, $54 \%$ am zweiten und $52 \%$ am dritten Testtag. Damit ist wahrscheinlich, daß die Ergebnisse des Erinnerungstestes das Erinnerungsvermögen der Tiere belegen. Dem Erinnerungstest folgte unmittelbar ein Umlerntest auf die Farben Rot und Blau. Der Abfall des Anteils richtiger Standbesuche in den ersten zwei Tagen gegenüber den vorausgegangenen Tests könnte durch die neue und möglicherweise schwierigere Aufgabe bedingt sein. Am 121. Lebenstag begann ein zweiter Umlerntest. Die Kälber sollten die Buchstaben A und O im Sinne einer Musterdifferenzierung unterscheiden. Auch bei dieser Aufgabe erreichten die Kälber bereits ab 3.Tag Erfolgsraten zwischen 60-70\%.

\section{Diskussion}

Die Ergebnisse zur Variabilität der Aktivität der Kälber, gemessen an der Anzahl Standbesuche je Tier und Tag (Abb. 2), decken sich mit den Beobachtungen für andere, quantitative Merkmale. Die Unterschiede zwischen den Individuen sind beträchtlich und unterstreichen die Notwendigkeit zur Prüfung repräsentativer Stichproben. Entsprechende Ableitungen zur statistischen Versuchsplanung von Lernexperimenten wurden von NÜRNBERG und FRANZ (1997) vorgestellt.

Aus dem Versuch 3 zur Einschätzung eventueller unerwarteter Erkennungsmöglichkeiten für die Stände, in denen eine Belohnung zu erhalten war ( $\mathrm{S}+$ ) durch die Tiere, z.B. durch den Geruch dieser Stände oder an den Sauggeräuschen anderer Kälber, ist zu schließen, daß die Nutzung solcher Erkennungsmöglichkeiten bei der gegebenen Situation nicht wahrscheinlich ist. Aus der Abbildung 4 wird deutlich, daß die Kälber auch nach der Veränderung des S+ Stimulus am 29. Tag zu 70 - $80 \%$ die Stände besuchten, die mit dem bisherigen $\mathrm{S}+\mathrm{Stimulus}$ gekennzeichnet waren, obwohl sie mögliche Vorbilder bei deren Milchaufnahme in den Ständen mit dem früheren S- Stimulus beobachten konnten (vgl. FRANZ u.a. (1995)).

Die in den vier Tests ermittelten Lernerfolge der Kälber stehen in guter Übereinstimmung mit den Ergebnissen von SCHAEFFER und SIKES (1971), von BALDWIN (1981) und von ARAVE u.a. (1992).

Danach sind Kälber in der Lage, verschiedene visuelle Differenzierungsaufgaben innerhalb weniger Tage mit Erfolg zu lösen.

Die vorgestellten Ergebnisse wurden an Kälbern ermittelt, die, im Unterschied zu den bisher publizierten Ergebnissen anderer Autoren, in kleinen Gruppen geprüft wurden. Von 33 Autoren (s. Tab. 1) führten nur zwei ihre Experimente mit in Gruppen gehalte- 
nen Rindern durch. Der Einzeltest von Kälbern, Jungrindern oder Kühen dominierte bisher.

Auch bei den Lernexperimenten mit Zoo- und Labortieren stellen Gruppentests bis heute sehr seltene Ausnahmen dar. Beispiele für solche Experimente lieferten STAMMBACH (1988), WASHBURN u.a. (1994) und MEIER u.a. (1998). STAMMBACH (1988) beobachtete Gruppen von 7 - 9 Javaneraffen (Macaca fascicularis), in denen bestimmte Tiere vorher so trainiert waren, daß sie einen speziellen Futterautomaten bedienen konnten. Dafür waren drei Hebel in einer bestimmten Sequenz zu ziehen. WASHBURN u.a. (1994) untersuchten mit Rhesusaffen (Macaca mulatta), ob deren Aktivität bei der Benutzung eines Joysticks zur Erlangung bestimmter, computergestützter Stimuli durch die Haltungsbedingungen (Einzelhaltung versus paarweise Haltung ) beeinflußt wird. MEIER u.a. (1998) entwickelten für visuelle Differenzierungsaufgaben eine automatisierte Trainingsapparatur für Gerbils (Meriones unguiculatus ) in Gruppenhaltung. Die Tiere konnten durch eine von zwei Türen, die durch visuelle Stimuli gekennzeichnet waren, zu einem Futterplatz gelangen, an dem sie bei Richtigwahl eine Futterportion erhielten.

Die für Labortiere hauptsächlich eingesetzten Lernapparaturen, wie die „Skinner Box“, das „Morris Water Maze“, verschiedene Labyrinthe und Wahlanlagen lassen die Nutzung mit Tiergruppen kaum zu. Um sozial lebende Tiere in der für sie typischen Gruppensituation hinsichtlich ihrer sensorischen oder kognitiven Fähigkeiten prüfen zu können, erscheint die Entwicklung dafür besser geeigneter Methoden erforderlich und auch möglich.

Als Störfaktor für Prüfungen in der Gruppensituation kann eine gegenseitige Beeinflussung der Tiere bezüglich des spezifischen Lerntestes auftreten. Sowohl eine Behinderung als auch eine Hilfe bezüglich des jeweiligen Lernparadigmas sind störende Einflüsse und sollten bei den beschriebenen Experimenten zur Entwicklung der Methode sowie der Beurteilung der Ergebnisse berücksichtigt werden.

Die Vorbildwirkung kann bei einer Gruppenprüfung von Tieren in dieser Anlage nicht ausgeschlossen werden. Da in der für Nutztierhaltungen typischen Gruppenhaltung mögliche Vorbildwirkungen für die meisten natürlichen Lernsituationen in der Regel gegeben sind, ist die Entscheidung über die Notwendigkeit ihrer Elimination von Fall zu Fall zu treffen.

Die entwickelte und oben beschriebene Mehrkanalwahlanlage für Kälber kann für die Prüfung des Wahlverhaltens einzelner Tiere und von kleinen Tiergruppen $(n=2-6)$ empfohlen werden. Dabei ist die freie Wahlmöglichkeit für jedes beteiligte Tier und die bedarfsgerechte Versorgung mit Futter und Tränke zu gewährleisten.

\section{Literatur}

ALBRIGHT, J.L.; GORDON, W.P.:

Behavioral responses of cows to auditory training. J. Dairy Sci., Champaign. Ill. 49 (1966), 104-106

ARAVE, C.W.; DOBSON, D.C.; ARAMBEL, M.J.; BOMAN. R.L.; GIILBERT. B.J.:

Value of a masking agent or feed flavor in enhancing preference for concentrate with added processed chicken excrete. J. Dairy Sci., Champaign, Ill. 68 (1985), 159 
ARAVE, C.W.; ARAMBEL, M.J.; PURCELL D.; WALTERS, J.L.:

Effect of alfalfa hay curing method on preference by Holstein heifers. J. Dairy Sci., Champaign, IIl. 71 (1988), 251

ARAVE, C.W.; STEWART, P.H.; WEBER, T.E.; BARKER, B.:

Use of feed flavors to enhance palatability of an already palatable dairy concentrate. J. Dairy Sci., Champaign, Ill. 73 (1990), 237

ARAVE, C.W.; ARAMBEL, M.J.; STEWART, P.; WEBER, T.:

Effect of added concentrated separator by-product dried beet pulp on Holstein heifer preference for dried beet pulp. J Dairy Sci., Champaign, Ill. 74 (1991), 190

ARAVE, C.W.; LAMB, R.C.; ARAMBEL, M.J.; PURCELL, D.; WALTERS, J.L.:

Behavior and maze learning ability of dairy calves as influenced by housing, sex and sire. Appl. Anim. Behav. Sci. 33 (1992), 149-163

ARAVE, C.W.; STEWART, P.H.; HANSEN, A.L.T.; WALTERS, J.L.:

ARAVE, C.W

Primary color discrimination by Holstein heifers. Proc W Sect Am Soc Anim. Sci. 44 (1993), 113

Assessing sensory capacity of animals using operant technology. J. Anim. Sci., Albany, N.Y. 74 (1996), 1996-2009

BALDWIN, B.A.:

Shape discrimination in sheep and calves. Anim. Behav. 29 (1981), 830-834

BOISSY, A.; LENEINDRE, P.:

Social influences on the reactivity of heifers: implications for learning abilities in operant conditioning. Appl. Anim.Behav. Sci. 25 (1990), 149-165

DABROWSKA, B.; HARMATA, W.; LENKIEWICZ, Z.; SCHIFFER, Z.; WOJTUSIAK, R.J.: Colour perception in cows. Behav. Proc. $6(1981), 1$

ENTSU, S.; DOHI, H.; YAMADA, A.:

Visual acuity of cattle determined by the method of discrimination learning. Appl. Anim. Behav. Sci. 34 (1992), 1-10

FRANZ, H.; SCHMIDT, H.P.; SIEBERT, K.; RÄDER, I.; PAPSTEIN, H.J.:

Learning and memory in cattle. Proc 23 rd Neurobiology Conference (Göttingen), Georg Thieme Verlag, Stuttgart, New York II: 234 (1995) (Abstract)

GILBERT, B.J.; ARAVE, C.W.:

Ability of cattle to distinguish among different wavelengths of light. J. Dairy Sci., Champaign. Ill. 69 (1986), 825-832

GRAMLING, G.E.; JENSEN, E.L.; WIECKERT, D.A.:

GRANDIN, T.: Learning ability in dairy calves. J. Dairy Sci., Champaign, Ill. 53 (1970), 659

The reluctance of cattle to change a learned choice may confound preference tests. Appl. Anim. Beh. Sci. 39 (1994), 21-28

GRÄF, R.; SAMBRAUS, H.H.:

Untersuchungen über das Farbsehvermögen von Rindern. Tierärztliche Umschau 33 (1978), 597-583

HEFFNER, R.S.; HEFFNER, H.E.:

Hearing in large mammals: Horses (Equus caballus) and cattle (Bos taurus). Behav. Neuro. Sci. 97(1983), 299

HOONHOUT, F.J.:

An investigation into the hearing abilities of dairy cows. M. Soc. Sci. Thesis (1984), Uni Waikato, N Z

KILGOUR, R.:

The contributions of psychology to a knowledge of farm animal behaviour. Ruakure Animal Research Centre, Private Bag, Hamilton (New Zealand) 2 (1976), 197-205

KILGOUR, R.:

Use of the Hebb-Williams closed-field test to study the learning of Jersey cows. Anim. Behav. 29 (1981), 850-860

KILGOUR, R.:

Learning and the training of farm animals. Food Animal Practice 3 (1987), 269-284 
KILGOUR, R.; FOSTER, T.M.; TEMPLE, W.; MATTHEWS, L.R.; BREMNER, K.J.:

Operant technology applied to solving farm animal problems. An assessment. Appl. Anim. Behav. Sci. 30 (1991), 141-166

KLOPFER, F.D.; KILGOUR, R.; MATTHEWS, L.R.:

Paired comparison analysis of palatabilities of twenty foods to dairy cows. Proc. NZ Soc. Anim. Prod. 41 (1981), 242

KOVALCIK, K.; KOVALCIK, M.:

Learning ability and memory testing in cattle of different ages. Appl. Anim. Sci. 15 (1986), 27-29

KUDRYAWTZEW, A.A.:

Higher nervous activity and the physiology of the senses in lactating cows.Intern. Dairy Congr.,16(D)

LAUBE, R.B.: (1962): 565

Untersuchungen zum visuellen Wahrnehmungsvermögen der Milchkithe als Voraussetzung zur Entwicklung von Signalsystemen für eine prozeßgesteuerte Milchviehanlage. Universität Leipzig, Diss., 1975

LIEBENBERG, O; LAUBE, R.B.; PORZIG, E.:

Einige Mitteilungen über historisch bedeutsame ethologische Beobachtungen am Rind. (Bos taurus L.). Arch. Tierz., Berlin 14 (1971), 109-127

MEIER; M.; REINERMANN, R.; WARLICH, J.; MANTEUFFEL, G.:

An automated training device for pattern discrimination leaming of group-housed gerbils. Physiology \& Behavior, 63 (1998), 497 - 498

NÜRNBERG, G.; FRANZ, H.:

Modelling learning success in calves. Proc. 48 th Annual Meeting EAAP (1997), 25 -28 8 , Vienna (Abstract)

PIOTROWSKI, J.:

Wahlversuche. Persönliche Mitteilung (1992), (Abstract)

POLTEN, S.:

Untersuchungen zum Wahrnehmungsvermögen der Milchkühe bezüglich Erkennung akustischer Signale, ein Beitrag zur Entwicklung von Signalsystemen für eine prozeßgesteuerte Milchviehanlage. Universität Leipzig, Diss., 1974

PORZIG, E.; LAUBE, B.; HENKE, M.:

Einflüsse auf das Anpassungsverhalten der Tiere durch Lernen. Arch. Tierz., Berlin 21 (1978), 220-237

PORZIG, E.; POLTEN, S.; LIEBENBERG, O.:

Untersuchungen von akustischen Reizen in ihrem stimulierenden Einfluss auf die Lokomotorik von Rindern. Arch. Tierz., Berlin 22 (1979), 299-307

PURCELL, D.:

Effects of four types of housing on growth and behaviour of split-embryo calves. M S Thesis, Utah State Univ. (1988), (Abstract)

PURCELL, D.; ARAVE, C.W.:

Isolation vs. group rearing in monozygous twin heifer calves. Appl. Anim. Behav. Sci. 31 (1991), 147-156

RIOL, J. A.; SANCHEZ, J. M.; EGUREN, V. G.; GAUDIOSO, V. R.:

Colour perception in fighting cattle. Appl. Anim. Behav. Sci. 23, (1989), 199

SCHAEFFER, R.G.; SIKES, J.D.:

Discrimination learning in dairy calves. J. Dairy Sci., Champaign, III. 54 (1971), 893-896

SOFFIE, M.; THINES, G.; FALTER, U.:

Colour discrimination in heifers. Mammalia 44 (1), (1980), 97

STAMMBACH, E.:

Group responses to specially skilled individuals in a macaca fascicularis group. Behaviour, 107 (1988), 241-266

THINES, G.; SOFFIE, M.:

Preliminary experiments on colour vision in cattle. British-Veterinary-Journal 133 (1977), 97-98

WANDER, I.F.:

Haltungs- und verfahrenstechnisch orientierte Verhaltensforschung. Züchtungskunde, Stuttgart 48 (1976), 447-459 
WARNICK, V.D.; ARAVE, C.W.; MICKELSEN, C.H.:

Effects of group, individual, and isolated rearing of calves on weight gain and behavior. J. Dairy Sci., Champaign, Ill. 60 (1977), 947-953

WASHBURN; D. A.; HARPER, S.; RUMBAUGH, D.M.:

Computer-task testing of rhesus monkeys (Macaca mulatta) in the social milieu. Primates 35 (1994), 343-351

WIECKERT, D.A.; JOHNSON, L.; OFFORD, K.P.; BARR, G.R.:

Measuring learning ability in dairy cows. J. Dairy Sci., Champaign, Ill. 49 (1966), 729

WIEDMEIER, R.D.; ARAVE, C.W.; ARAMBEL, M.J.; KENT, B.A.; WALTERS, J.L.:

Effects of concentrated separator dried beet pulp on nutrient digestibillity, milk production, and preference of Holstein Cattle. J. Dairy Sci., Champaign, III. 77 (1994), 3051-3057

Eingegangen: 11.06 .1998

Akzeptiert: 23.02.1999

Anschrif des Verfassers

Dr. HARTMUT FRANZ

Forschungsinstitut für die Biologie landwirtschaftlicher Nutztiere

Forschungsbereich Physiologische Grundlagen der Tierhaltung

Wilhelm-Stahl-Allee 2

D-18196 Dummerstorf

E-Mail: franz@fbn-dummerstorf.de 